\title{
Effects of Modeling Method on Prediction of Electronic Properties of Carbon Nantobues and Silicon Nanotubes
}

\author{
Shuang $\mathrm{Xu}^{\mathrm{a}}$, Wu-Gui Jiang ${ }^{\mathrm{b}}$, Qiu-Wei Fu ${ }^{\mathrm{c}}$, Ren-Zhi Zhong ${ }^{\mathrm{d}}$, Min Wei ${ }^{\mathrm{e}}$ \\ School of Nanchang, Hangkong University, Nanchang 330063, China;

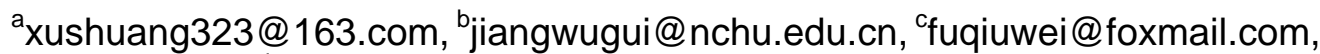 \\ dlukerenzhi@126.com, ${ }^{e}$ minwei_1126@163.com
}

Keywords: Armchair; DOS; HOMO-LUMO; Electronic properties; DFT

\begin{abstract}
Based on the density functional theory (DFT), we predict electronic properties of the non-periodic structure and the periodic structure of carbon nanotubes (CNTs) and silicon nanotubes (SiNTs). In the simulation process, we studied how to increase the length of nanotubes or add $\mathrm{H}$ atoms to dangling bond to describe the feasibility of the non-periodic structure. The results show that, for the non-periodic structure of CNTs, compared with only increasing the length of the nanotubes, the simulation result of adding $\mathrm{H}$ atoms to dangling bond is better. For the non-periodic structure of SiNTs, the simulation result of increasing the length is more reasonable.
\end{abstract}

\section{Introduction}

Since the discovery of carbon nanotubes (CNTs) in 1991[1], because of the unique cylinder structure consisting of a graphene sheet, its electronic and structural properties have attracted many attentions [2-6]. A lot of theoretical and experimental studies showed that the electronic properties of nanotubes are mainly determined by the chirality and diameter of nanotube. The band structure calculation results show that single-walled carbon nanotubes have a conductor and semiconductor properties, which has been confirmed by experiment [7-10]. Part of single-walled silicon nanotubes were studied by using first-principles method and molecular dynamics method [11-15]. Effect of doping on the structural and electronic properties of silicon nanotubes were also studied [16-19].Seifert et al studied the electronic properties of the periodic structure of si-based nanotubes,the results showed the Si-based nanotubes have a semiconducting gap, which in contrast to carbon nanotubes is largely independent of the tube diameter and chirality[20]. S.B. Fagan et al studied the electronic properties of the periodic structure of SiNTs, the results showed, depending on their chiralities and diameters, the silicon nanotubes may present metallic (armchair) or semiconductor (zigzag and mixed) behaviors[21].Thus, modeling method has a significant influence on the prediction of the electronic properties for nanotubes.

The main purpose of this paper is to predict the effects of modeling method on the electronic properties of CNTs and SiNTs, in order to try to establish the relationship between the periodic structure of nanotubes and the non-periodic structure of nanotubes.

\section{Calculation method and Models}

Calculation method. DFT is the functinoal which energy is regarded as system's particle density. It is a calculation method developed by the Walter-Kohn. It is unnecessary to solve complex wave function with 3n electronic coordinates by using DFT, which simplify the problem. The Kohn-sham DFT energy expression is,

$$
E_{V}[\delta]=\int V(r) \rho(r) d r+\frac{1}{2} \iint \frac{\rho(r) \rho\left(r^{\prime}\right)}{\left|r-r^{\prime}\right|} d r d r^{\prime}+T[\rho]+E_{e x c}[\rho]
$$

Where, the first item is attraction potential between nuclear and electron, the second item is the electrotatic interaction energy, the third item $T[\rho]$ is the knetic energy, the forth item $E_{\text {exc }}[\rho]$ is echange-correlation energy. When $\rho(r)$ slowly changes, $E_{e x c}[\rho]$ can be expressed as, 


$$
E_{e x c}[\rho] \approx \int \rho(R) \varepsilon_{E X C}[\rho(r)] d r
$$

where, $\varepsilon_{E X C}$ is electronic exchange-correlation energy, $\phi i(r)$ is obtained by sloving the Schrödinger equation, the expression of $\rho(r)$ is as follows,

$$
\rho(r)=\sum|\phi i(r)|
$$

Calculation models. DOS is calculated to analyze the band gap characteristics, if the Fermi level in the DOS value is zero, the system is a semiconductor or insulator, if there is a wave of DOS across the Fermi level, the system is a metal. HOMO-LUMO calculations are performed to investigate whether a molecule is easy to motivated. The function PWC belonging to the local density approximation (LDA) is used to process the exchange correlation potential energy.The orbit population parameter smearing is set at $0.1 \mathrm{Ha}$. The periodic structure model is shown in fig.1. The non-periodic structure of nanotubes are obtained by removing the periodic boundry. The model of adding $\mathrm{H}$ atoms to dangling bond nanotubes is built, structure diagram of different pespective of the non-periodic structure of adding $\mathrm{H}$ atoms to dangling bond are respectively shown in fig.2.

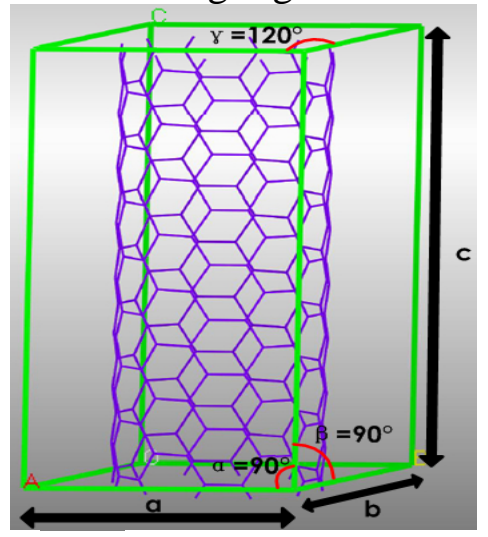

Fig. 1 The periodic structure model

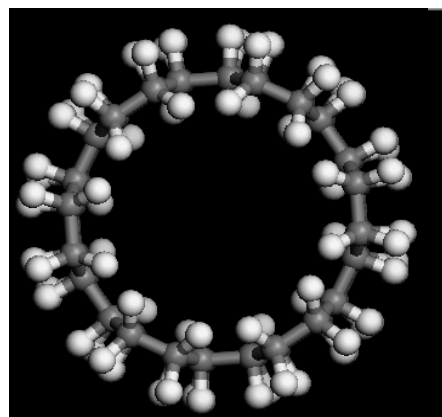

Fig.2(a) Sectional view

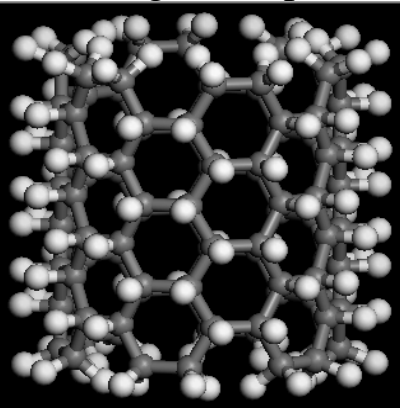

Fig.2(b) Front view

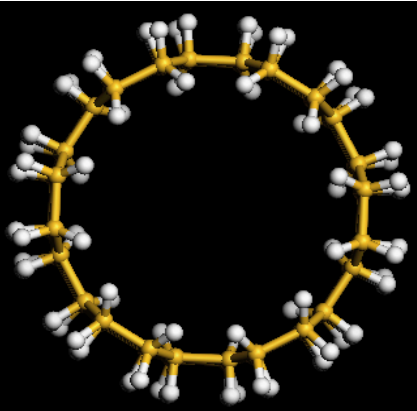

Fig.2(c) Sectional view

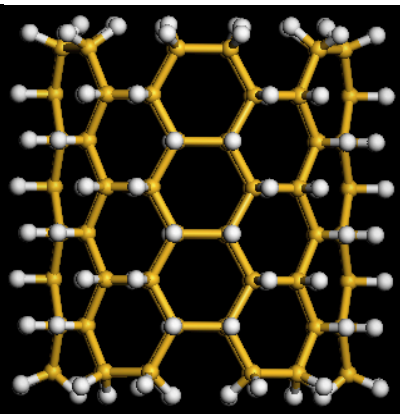

Fig.2(d) Front view

\section{Simulation results and discussion}

Effects of different repeat units on the stability of the periodic structure of CNTs and SiNTs. The supercells are built, $a, b$ is constant, $c$ is variable, in order to make the periodic structure of nanotubes with different repeat units. It can be seen from Fig.3(a) and Fig.3(b), when repeat units is 8,the value of DOS is located on the line instead of the peak at the Fermi energy, the system is relatively stable. 


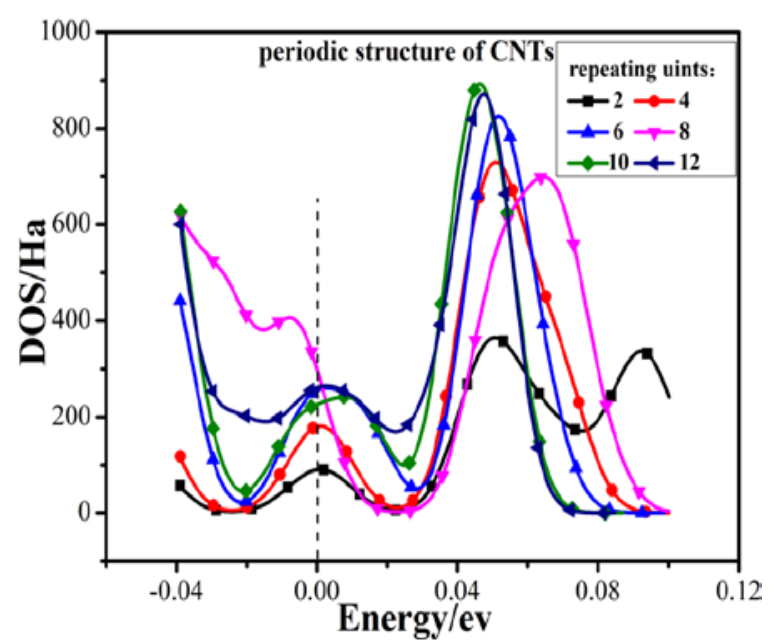

Fig.3(a) DOS of CNTs

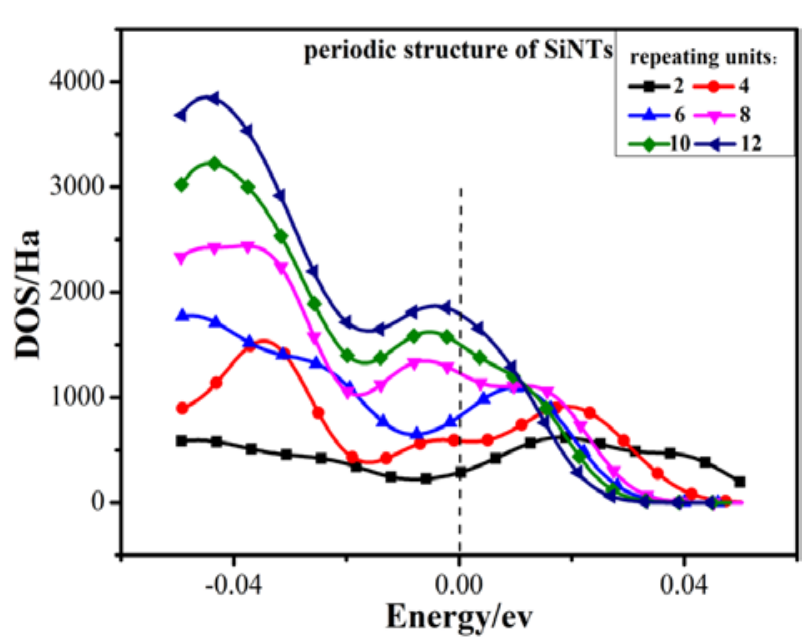

Fig.3(b) DOS of SiNTs

Effects of length and adding $\mathrm{H}$ atoms to dangling bond on prediction of the electronic properties of the non-periodic structure of CNTs. It can be seen from Fig.3(c), after repeat units reach to 8,the curve of DOS is gradually to be regular. We can also see from Fig.3(d), the edge of nanotubes is neutralized after adding $\mathrm{H}$ to dangling bonds, the curve of DOS is more regular. The value of DOS is gradually close to the trough with the increase of length, the system is more stable.

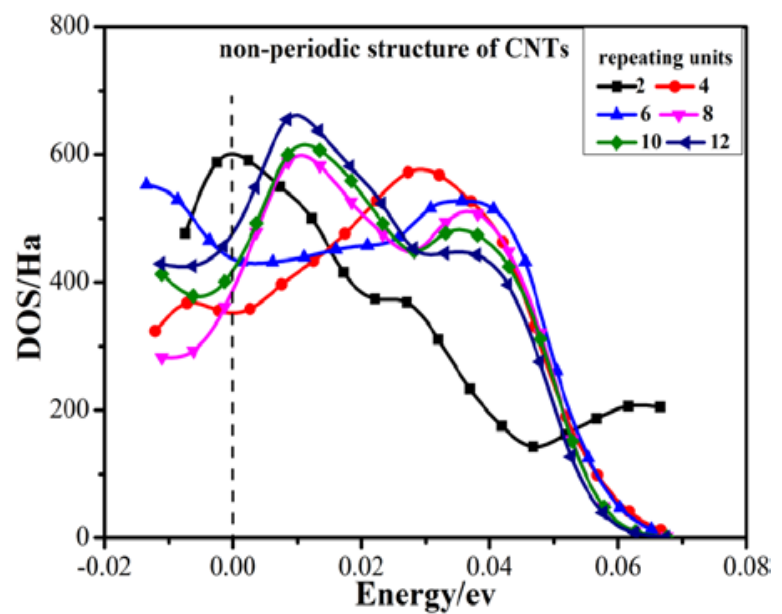

Fig.3(c) DOS of increasing the length

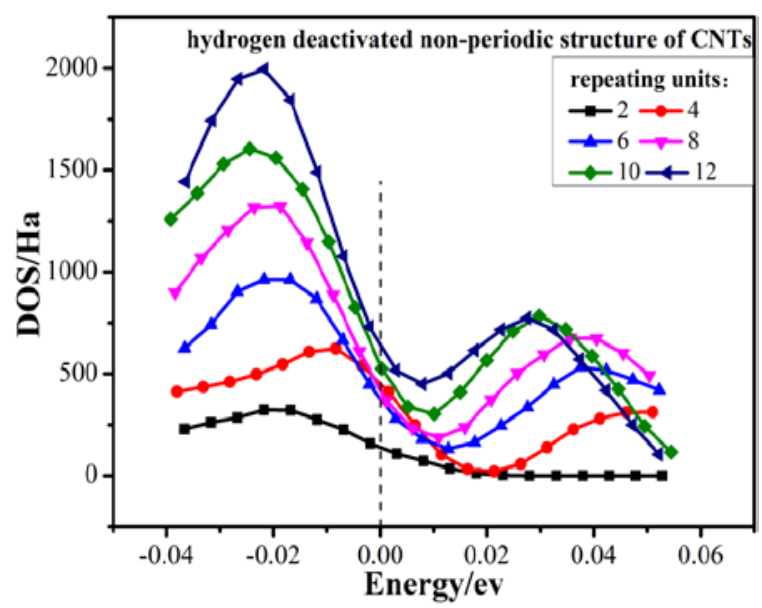

Fig.3(d) DOS of adding $\mathrm{H}$ atoms to dangling bond

It can be seen from Fig.3(e), the value of HOMO-LUMO of the periodic structure of CNTs is greater than zero. Although it is positive, but the value is very small, it turns out to be metallic.For the non-periodic structure of CNTs, the value of HOMO-LUMO is smaller than zero, which is obvious to be metallic. The value of HOMO-LUMO with two different modeling methods is gradually close to the periodic structure, but the simulation result of adding $\mathrm{H}$ atoms to dangling bond is better. 


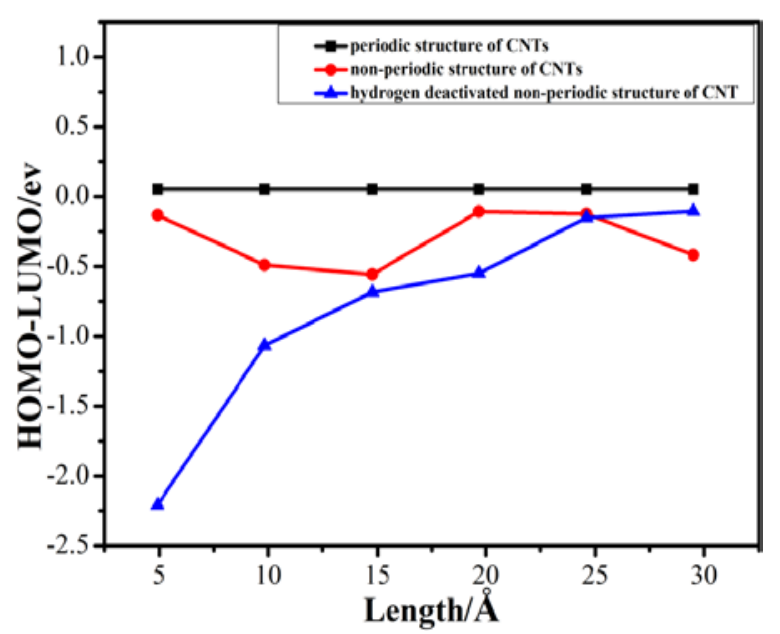

Fig.3(e) HOMO-LUMO of increasing the length

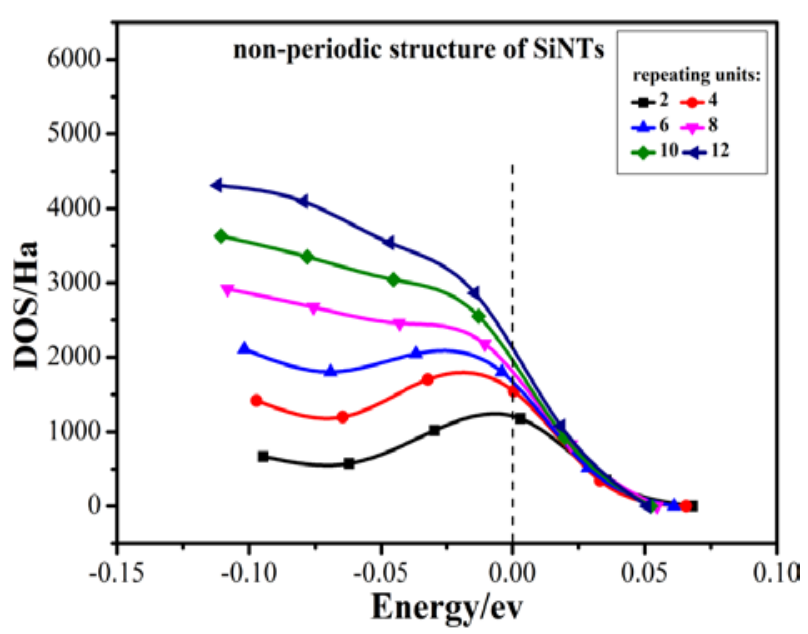

Fig.3(f) DOS of increasing the length

Effects of length and adding $H$ atoms to dangling bond on prediction of the electronic properties of the non-periodic structure of SiNTs. The curve of DOS on the basis of increasing the length of SiNTs is shown in the Fig.3(f). The curve of DOS on the basis of adding $\mathrm{H}$ atoms to dangling bond is shown in the Fig.3(g). The curve of HOMO-LUMO on the basis of increasing the length of CNTs is shown in the Fig.3(h). It can be seen from Fig.3(f) and Fig.3(g), compared with CNTs, the curve of DOS of SiNTs is relatively regular. the value of DOS with the increasing of length shifts gradually from the peak to the trough at the Fermi position, which shows the system of SiNTs is more stable. It can be seen from Fig.3(h), Compared with adding $\mathrm{H}$ atoms to dangling bond, the simulation result of increasing the length of the nanotubes is better. Compared Fig.3(e) and Fig.3(h), we can conclude that the simulation result of CNTs and SiNTs is contrary.

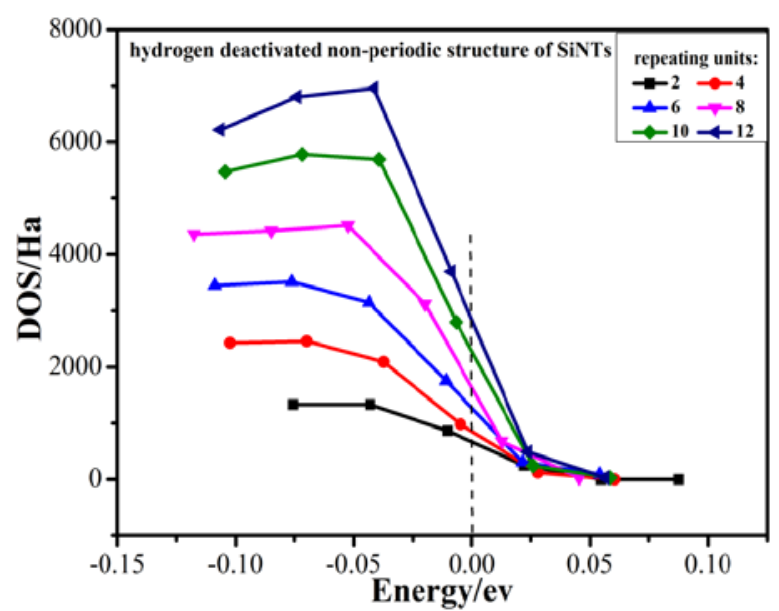

Fig.3(g) DOS of adding $\mathrm{H}$ atoms to dangling bond

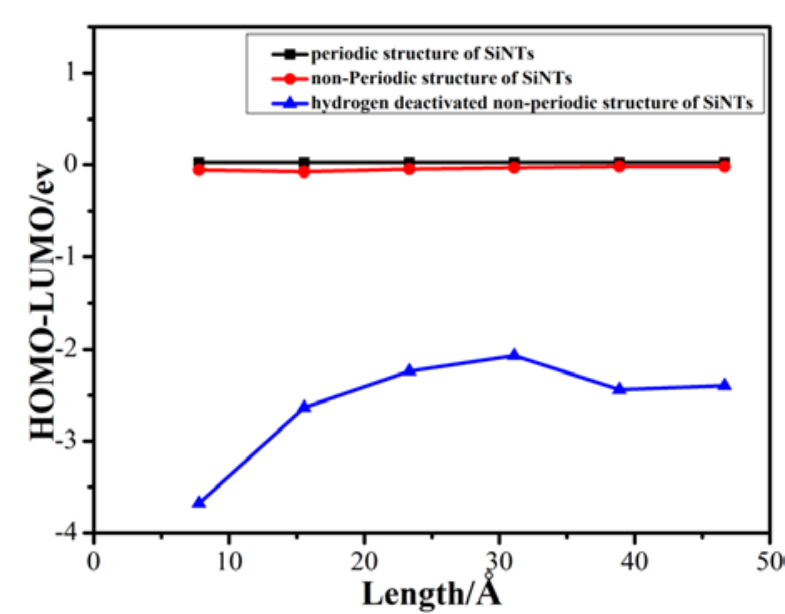

Fig.3(h) HOMO-LUMO of increasing the length

\section{Summary}

In this paper, DOS and HOMO-LUMO are calculated to predict electronic properties of carbon nanotubes (CNTs) and silicon nanotubes (SiNTs). The results show that, for the non-periodic structure of CNTs and SiNTs, the system is more and more stable with the increase of the length of nanotubes, and the electronic properties are gradually close to the periodic structure. For CNTs, compared with only increasing the length of the nanotubes, the simulation result of adding $\mathrm{H}$ atoms to dangling bond is better. For SiNTs, the simulation result of increasing the length of the nanotubes is more reasonable. 


\section{Reference}

[1]S. Iijima, Helical microtubes of graphitic carbon, J. Nature. 354 (1991) 56-58.

[2]G. Bertoni, L. Calmels, First-principles calculation of the electronic structure and energy loss near edge spectra of chiral carbon nanotubes, J. Micron. 37 (2006) 486-491.

[3]R. Nizam, S. Mahdi, A. Rizvi, A. Azam, Calculating Electronic Structure of Different Carbon Nanotubes and it's affect on Band Gap, J. International Journal of Science and Technology. 1(2011)153-162.

[4]R.S. Ruoff, D. Qian, W.K. Liu, Mechanical properties of carbon nanotubes:theoretical predictions and experimental measurements, J.Comptes Rendus Physique. 4 (2003) 993-1008.

[5]B.I. Yakobson, P. Avouris, Mechanical Properties of Carbon Nanotubes, J.Topics in Applied Physics. 80 (2001) 287-327.

[6]J.J. Zhao, H. Park, J, Han, J.P. Lu, Electronic Properties of Carbon Nanotubes with Covalent Sidewall Functionalization, J. Physical Chemistry B. 108 (2004) 4227-4230.

[7]K. Gharbavi, H. Badehian, Structural and electronic properties of armchair $(7,7)$ carbon nanotubes using DFT, J.Computational Materials Science. (2013).

[8]G.B. Abadir, K. Walus, D.L. Pulfrey, Basis-set choice for DFT/NEGF simulations of carbon nanotubes, J. Comput Electron. (2009)1-9.

[9]R. Satio, M. Fujita, G. Dresseihaus, Electronic Structure and Growth Mechanism of Carbon Tubes, J. Materials Science and Engineering. 19(1993) 185-191

[10]W.Z. Liang, S. Yokojima, E.NgM, Optical properties of single-walled 0.4nm Carnon nanotubes, J. J. Am. Soc. 123 (2001) 9830-9836.

[11]Y. Zhu, Z.B. Qu, G.L. Zhuang, Coronal multi-walled silicon nanotubes, J. Journal of Energy Chemistry. (2013) 408-412.

[12]M. Zhang, Y.H. Kan, Q.J. Zang, Why silicon nanotubes stably exist in armchair structure? J. Chemical Physics Letters. (2003) 81-86.

[13]E.C. Anota, A.B. Hernández, M. Castro, Investigating the electronic properties of silicon nanosheets by first-principles calculations, J. Springer (2011).

[14]T. Qiu, X.L. WU, Y.F. Mei, From Si nanotubes to nanowires:Synthesis, characterization, and self-assembly, J. Journal of Crystal Growth. (2005).

[15]V. V. Filippow, A. A. Zavorotnii. Simulation of electronic properties of silicon nanotubes, J. Russian Physics Journal. (2013).

[16]A. Hever, J. Bernstein, O. Hod. Structural and electronic properties of sp3 type silicon nanotubes, J. The journal of chemical physics. 21 (2012) 137.

[17]M .Menon, A. Andriotis, G. Froudakis, Structure and stability of Ni-encapsulated Si nanotube, J. Nano Letters. 2 (2002) 301-304.

[18]K .A. Singh, T.M. Briere, V. Kumar, Magnitism in transition-metal-doped silicon nanotube, J. Physical Review Letters. 91 (2003) 1-4.

[19]L.J. Guo, X.H Zheng, C.S. Liu, An ab initio study of cluster-assembled hydrogenated silicon nanotubes, J.Computational and Theoretical Chemistry. (2011).

[20]G. Seifert, T. Kohler, H. M. Urbassek, Tubular structures of silicon[J]. Physical Review B. (2001).

[21]S.B. Fagan, R. Mota, R.J. Baierle, Stability investigation and thermal behavior of a hypothetical silicon nanotube, J. Journal of Molecular Structure. (2000). 